\title{
Pico-current Measurement Challenges and Remedies: A Review
}

\author{
Abdul Al Mortuza ", Md. Firoz Pervez, Md. Khalid Hossain, Sapan Kumar Sen, \\ Md. Nasrul Haque Mia, M. K. Basher, Md. Shah Alam
}

Institute of Electronics, Atomic Energy Research Establishment, Bangladesh

Copyright $(2017$ by authors, all rights reserved. Authors agree that this article remains permanently open access under the terms of the Creative Commons Attribution License 4.0 International License

\begin{abstract}
A Pico-ammeter has versatile application in the measurement of low current of nuclear detector, biological cell or tissues and to know the character of new electronic devices. But noise can be generated due to some internal and external sources such as, poor design, placement of ammeter, dust, humidity, bending of connecting wires and even the selection of electronic devices, thus the measurement goes tricky. Several design techniques, guarding, shielding, and an unconventional wiring technique can help to measure low current accurately by reducing noise. This paper describes this low current measurement problems in detail and their corresponding solutions. It also shows the comparison of different low current measurement systems (LCMS). Feedback type ammeters are more preferable than shunt ammeter and current conveyor technique. In operational amplifier selection, CMOS type op-amp is better than BJT and FET.
\end{abstract}

Keywords Pico-current, Triboelectric Effect, Guarding, Shielding, LCMS

\section{Introduction}

Ammeters are commonly connected in series to measure current flow through the circuit. But it does not seem to us an important matter the placement an ammeter in a circuit i.e., in-between load and source or after load to ground. Placement of an ammeter does not make any effect on the current source for typically $>1 \mathrm{~mA}$, but it plays an important role for an ultralow current (pA to fA range) measurement. In every cases load has to be placed in-between the ammeter and source, not to the ground [1]. As a simple matter of placement of an ammeter has an effect on measurement, special design and setup is required for ultralow current measurement.
Picoammeters have versatile field of applications in research and engineering such as radioactive measurement, biomedical, chemistry, electrochemistry, magnetism and to discover electronic device character [2,3]. In nuclear physics Geiger Muller tubes, photo multiplier tubes, accelerometers, photo detectors and other type of sensors produce very low current in the range from $10^{-7}$ to $10^{-13} \mathrm{~A}$ depending on the activity of radionuclide [4].

Picoammeter is also applicable to measure leakage current of capacitors and transistors, reverse leakage current of high voltage diodes, gate leakage current and sub-threshold current of MOSFETs [5,6]. Gamma and photon measurements with photomultiplier tubes, ion beam measurements, and I-V characteristics of carbon nanotube (CNT), wafer level photo diode testing and photoconductive current measurement of PIN photodiode are also its application [5]. In bio-sensor research, picoammeter is applicable for clinical diagnosis, DNA probing and monitoring systems $[7,8]$.

In general, current is directly measured, i.e., current is the prime concern to measure current only but now voltage is also one of the important parameter which can be measured to calculate current [9]. There is no better design available for measuring nano and pico range current; moreover, noisy background makes an effect on measurement [7]. Typically, Two basic circuit designs based on operational amplifier are available to measure low current; these are the shunt ammeter circuit and the feedback ammeter circuit $[4,5,10,11]$. The shunt amplifier circuit is commonly used in general purpose DMMs, where a small value resistor is used as a shunt and a small voltage drop across it called burden voltage [10] shown in figure 1. Considering the loading effect, shunt resistor is as low as preferable. Moreover, low value resistors have fast response time, higher temperature stability, and higher voltage coefficient than that of higher value resistor $[12,13]$. 


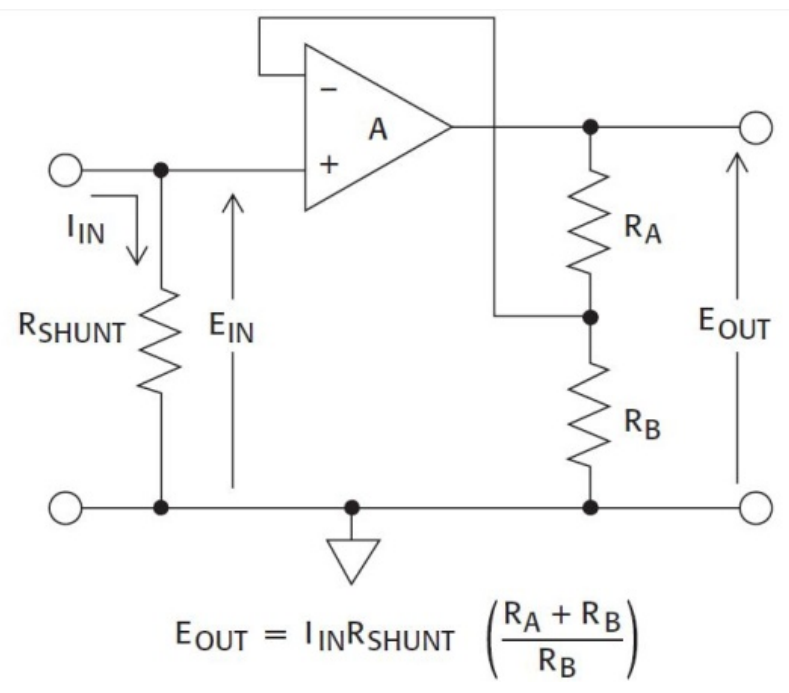

Figure 1. Shunt amplifier [12].

However, low burden voltage (in the range of about 200 $\mathrm{mV}$ to $2 \mathrm{~V}$ ) increases the Johnson noise [4][10]. Additionally, there is a limitation in measurement of current below $10^{-11} \mathrm{~A}$, due to more short response [4].

Thus, appropriate shunt resistor selection is often a good trade-off between noise and burden voltage. The output voltage depends on the relative value of feedback resistor, $\mathrm{R}_{\mathrm{A}}$ and input resistor, $R_{B}$ respectively is given by [12]:

$$
E_{\text {OUT }}=E_{I N}\left(\frac{R_{A}+R_{B}}{R_{B}}\right)=I_{I N} R_{S H U N T}\left(\frac{R_{A}+R_{B}}{R_{B}}\right)
$$

At a question of fast response in ultra-low current measurement the shunt ammeter is slower than that of the feedback ammeter [10]. Considering both the problem of speed and shunt value selection, feedback type pico-ammeter can overcome all the shortcomings of the shunt amplifier method [4]. Thus, pico-ammeters and newer electrometers are commonly designed with feedback amplifier configurations [12]. In feedback type ammeter, capacitive feedback or resistive feedback configuration are commonly designed [8][14].

Capacitive feedback ammeter is a current integrator with high gain and large bandwidth. The performance of capacitive feedback as low current measurement system (LCMS) can be measured separately with two phases of operation namely: reset and integration phase. In reset phase, input and output terminals are shorted by means of short the feedback capacitor's terminals and the op-amp configuration act as a voltage follower. In this case, the only source of noise is operational amplifier itself $[8,14]$.

During the integration phase, the switch is opened shown in figure-2 and the circuit act as an integrator where, initially the capacitor is uncharged and integrates the input current. The resulting output voltage is:

$$
V_{\text {out }}=\frac{1}{C_{F}} \int_{0}^{T} I_{\text {in }}(t) d t
$$



Figure 2. Low current measurement system with capacitor feedback configuration.

The resistive feedback amplifier is an active transimpedance amplifier (TIA) which converts the input current to a voltage reading [10].

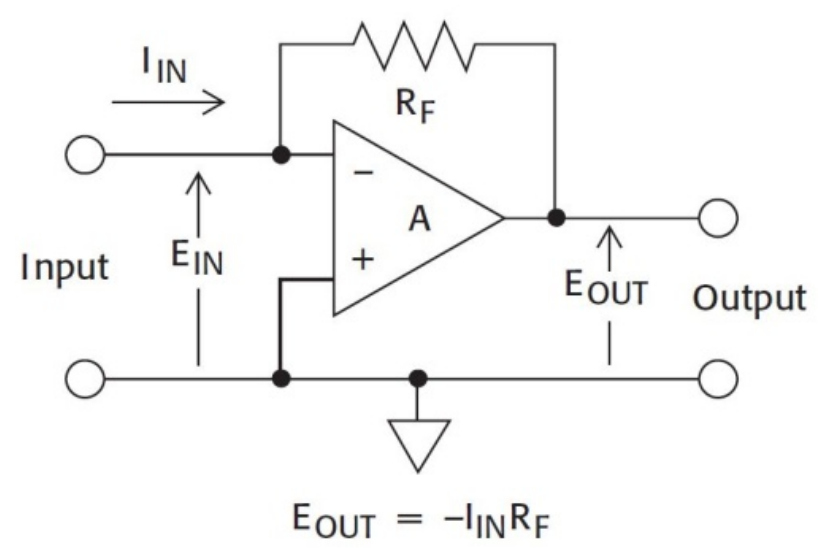

Figure 3. Feedback amplifier [12].

As op-amp of TIA has very high input impedance, very low offset voltage, and low burden voltage so the input current has a negligible change and the entire current would flow through the high feedback resistor (typically value $>10 \mathrm{G} \Omega$ ). In contrast, the Johnson noise current follows an inverse function with feedback resistor [4]. Feedback amplifier method has low voltage burden (in the range of $0.2-2 \mathrm{mV}$ ) and fast rise time can be achieved by using high gain operational amplifier [12].

Another approach is to measure low current called current conveyor technique that amplify the input current directly $[8,14]$. Current conveyor is a building block of current mode equivalent to operational amplifier perform in voltage mode with three (may have 4-6) terminals $(X, Y, Z)$ where, $X$ is low impedance terminal and $\mathrm{Y}, \mathrm{Z}$ are high impedance terminal $[15,16]$ shown in figure-4. The current conveyor was first introduced in 1968 but it was not coming in use at that time due lack of understand its applications. Now, it has been observed that the current conveyor has several advantages over operational amplifier, such as, higher voltage gains over a large bandwidth of a small signal $[15,17]$. In its operation if a voltage is applied in terminal Y, equal potential will appear 
at X. Similarly, an input current I in X terminal force to flow an equal amount of current (i.e., I) into the terminal Y. As well as, the current I will convey into the output terminal $\mathrm{Z}$ such as the output act as a current source with high input impedance. As the potential at $X$ is the same with that terminal $\mathrm{Y}$, the current conveyor can apply a clamping voltage to measure low currents [8]. Current conveyors are classified in three categories: first generation (CCI), second generation (CCII) and third generation (CCIII) respectively.

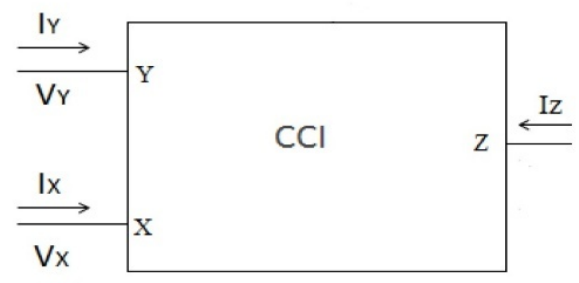

Figure 4. Block diagram of current conveyor.

The input and output relation of first generation current conveyor can be described with matrix equation as:

$$
\left[\begin{array}{l}
I_{Y} \\
V_{X} \\
I_{Z}
\end{array}\right]=\left[\begin{array}{ccc}
0 & 1 & 0 \\
1 & 0 & 0 \\
0 & \pm 1 & 0
\end{array}\right]\left[\begin{array}{l}
V_{Y} \\
I_{X} \\
V_{Z}
\end{array}\right]
$$

The current noise of the current conveyor (CCI) can be calculated as

$$
S_{I}=\left(8+\frac{2}{\alpha}\right)\left(4 K T^{2} \frac{2}{3} g_{m}+\frac{K_{F} I d^{A F}}{C_{O X}\left(L^{2}\right) f}\right)\left[A^{2} / H z\right]
$$

Where, $g_{m}$ is the transconductance of the input stage and $\alpha$ is the current amplification factor. In the CCI the overall noise is the sum of the noises at source current and the conveyor bias current. Therefore, the total noise is more than that of resistive feedback or capacitive feedback configuration. So, in low current measurement current conveyor circuits are not generally used. Moreover, D. Kim et al., reported that the capacitive feedback, resistive feedback and current conveyor technique can measure $750 \mathrm{fA}, 4 \mathrm{pA}$, and $600 \mathrm{pA}$ RMS at $10 \mathrm{kHz}$ respectively $[8,14]$.

\section{Sources of Noise}

There are several sources of noise during low current measurement that are, noise in source itself, interconnection, environmental condition and as well as design technique. The noise current is generated within the source itself is due to leakage, piezoelectric, or triboelectric effects, or through dielectric absorption $[9,10,12,13]$.

In resistive feedback system the dominant noise is the thermal noise of the feedback resistor and the input impedance of the current source. From the noise model of the resistive feedback system, the output power spectral density is given as:

$$
S_{V}=e_{R}^{2}+e_{M}^{2}\left(1+Y_{M} \cdot R_{F}\right)^{2}\left[V^{2} / \mathrm{Hz}\right]
$$

Where, $e_{M}$ is the combination of thermal and flicker noise of op-amp, $e_{R}$ is the thermal noise of the feedback resistance $\mathrm{R}_{\mathrm{F}}$ and $\mathrm{Y}_{\mathrm{M}}$ is the admittance of the equivalent input circuit (cells, tissue and ion chamber etc.).

In the capacitive feedback the output power spectral density at rest and integration phases are given as in equation (6) and (7) respectively:

$$
\begin{gathered}
S_{V, \text { rest }}=e_{M}^{2}\left[V^{2} / \mathrm{Hz}\right] \\
S_{V, \text { in }}=e_{M}^{2}\left(\frac{Y_{M}}{C_{F}}\left(1-e^{-T . s}\right)\right)^{2}\left[V^{2} / \mathrm{Hz}\right]
\end{gathered}
$$

Where, $C_{F}$ is the feedback capacitance and $\mathrm{T}$ is the integration time [14][20]. The measured noise spectral density for resistive feedback is around $2 \times 10^{-14}$, for capacitive feedback $1 \times 10^{-14}$, and for current conveyor $1.3 \times 10^{-12}[\mathrm{~A} / \sqrt{\mathrm{Hz}}]$ respectively for maximum bandwidth of $10 \mathrm{kHz}$. In higher frequency $(>10 \mathrm{kHz})$ capacitive and resistive feedback shows similar performance [21].

Noise can be also generated in the design circuit and semiconductor such as, thermal noise is generated due to rise of temperature of the electronic devices [22][23]. This type of noise is sometime called Johnson noise. It can be described by Nyquist equation:

$$
e_{R}^{2}=4 \mathrm{kT}
$$

where, $\mathrm{k}$ is the Boltzman constant, $\mathrm{T}$ is the absolute temperature in kelvin.

Noise associated with a discrete structure of electricity and the individual carrier injection through the $p n$ junction is known as shot noise [22,23]. The shot noise current is given by the equation:

$$
\mathrm{i}_{\mathrm{sh}}{ }^{2}=2 \mathrm{qI} \Delta \mathrm{f}
$$

Here, $\mathrm{q}$ is the electron charge, $\mathrm{I}$ is the forward junction current, and $\Delta f$ is the frequency change.

Random noise produced in all linear devices are known as $1 /$ f noise called flicker noise and is due to material failures or imperfection in the fabrication process. But the actual source of this noise is still unknown. It is the dominant source of noise in low frequency less than $200 \mathrm{~Hz}$ [22-24]. There are two models of $1 / \mathrm{f}$ noise, one is called surface model developed by McWhorter in 1957 used for MOSFET and another is bulk model by Hooge in 1968 for BJT devices [23]. Fluctuation of the number of carrier's generation-recombination at the centre of the junction of the transistor, there will be a noise called generation recombination noise. This type of noise is a function of temperature and biasing condition.

In triboelectric effect, charge is generated in between the insulator and conductor due to movement of conductor and insulator against each other. Here, free charge (electrons) rub off the conductor and create a charge imbalance that causes a current flow called triboelectric current. It is about hundreds of pA for polyethylene cable [19]. Excess triboelectric current can be minimized by reducing the length of the cable 
from source to ammeter. But this solution is not realistic due to less workability or less usability in measurement [25]. Coaxial or triaxial cable application can be an effective way to minimize this noise [26].

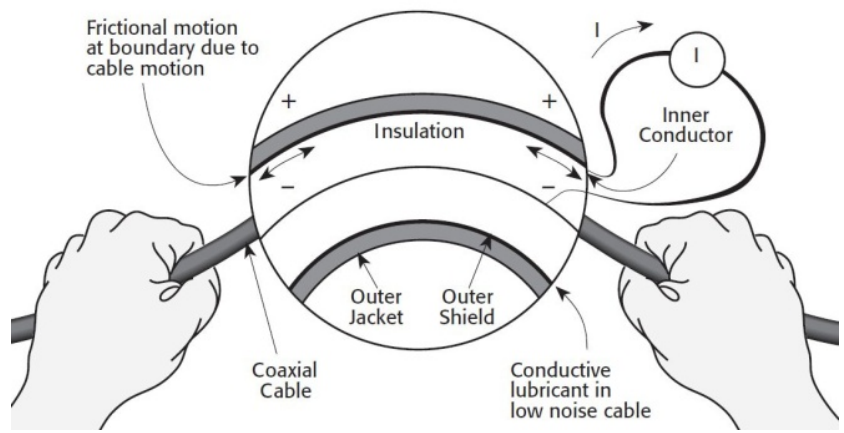

Figure 5. Triboelectric effect [5].

Piezoelectric currents can be generated in the insulating terminals and interconnecting circuits when mechanical stress is subjected to certain insulating material. So, special care should be taken to avoid unnecessary stress insertion to the circuit. Dirty PCB and other hydroscopic materials can absorb moisture, thus losses electrical resistance and leads to a leakage current between the conductors. Sometimes weak batteries are generated due to charge separation between two conductors on the circuit board, e.g., an epoxy printed circuit board may generate several nanoamperes current if etching solution, flux or other contaminations are not properly cleaned [12][27]. To overcome this problem, every interconnection of the circuit should be thoroughly cleaned with cleaning solvent such as isopropyl alcohol or methanol, and dry completely before use. But spry flux is not recommended for final cleaning because water soluble fluxes can leave a film behind [28]. Another way to reduce this piezoelectric current is using high surface resistivity and smaller dielectric absorption material such as, Teflon, Sapphire (resistivity $>10^{18}$ ) [5].

Electrical noise generated from the external environment greatly affects the low current measurement performance due to the capacitive coupling between environment and measurement setup [11].

Radio frequency (RF) or Electromagnetic frequency
(EMF) interference sources such as $50 / 60 \mathrm{~Hz}$ power line, $\mathrm{TV}$, radio broadcast, tube light, high voltage arcing and so on are the common sources of electrical noise. The human body can be an another external source of noise, as human body is normally charged and contains a certain level of potential, the coupling capacitance may induce noise current if one move here and there close to the signal line [11]. Light is also a source of electromagnetic noise because some components such as diodes, transistors are photo sensitive, thus light tight shielding is also required [5]. Metallic cage shielding is required to eliminate or minimize the external electromagnetic field and as well as desiccant packs can be used when humidity is an issue [27].

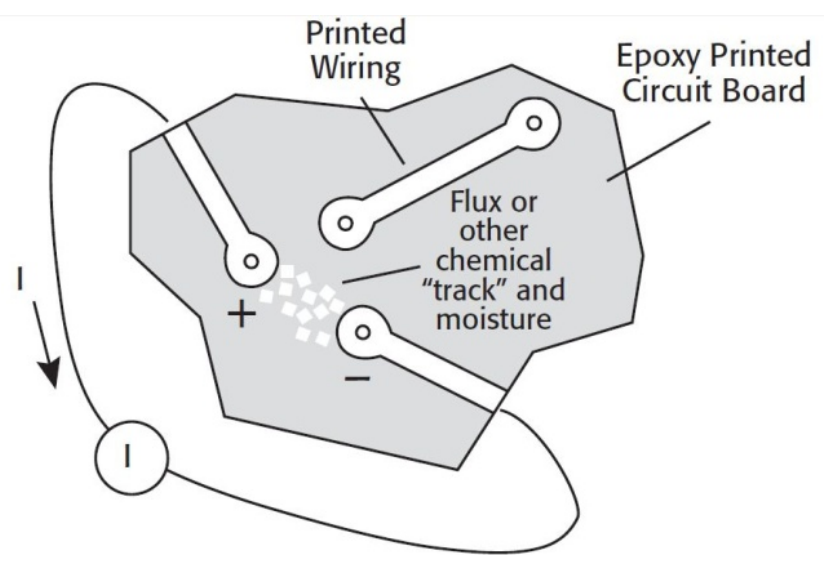

Figure 6. Electrochemical effect/ dielectric absorption effect [5].

\section{Design Technique}

Several design techniques and selection of components can improve the performance and accuracy of the pico-ammeter. In this regard, IC selection is an important part prior to designing ultra-low current measurement circuit. Input bias current, voltage and current noise and offset drift are due to temperature as low as preferable to design. A short list of comparison among three types of op-amp ICs are given in table 1.

Table 1. Comparison of different types of op-amp [24][29]

\begin{tabular}{|c|c|c|c|}
\hline Parameter & Bipolar & JFET & CMOS \\
\hline Input Offset voltage & $10 \mu \mathrm{V}-7 \mathrm{mV}$ & $500 \mu \mathrm{V}-15 \mathrm{mV}$ & $1-10 \mu \mathrm{V} /{ }^{\circ} \mathrm{C}$ \\
\hline Input offset voltage drift & $0.1-10 \mu \mathrm{V} /{ }^{\circ} \mathrm{C}$ & $5-40 \mu \mathrm{V} /{ }^{\circ} \mathrm{C}$ & $0.1-10 \mathrm{pA}$ \\
\hline Input Bias current & $100-50,000 \mathrm{pA}$ & $1-100 \mathrm{pA}$ & Doubles for every $10^{\circ} \mathrm{C}$ increase \\
\hline Input bias current drift & Fairly stable & Doubles for every $10^{\circ} \mathrm{C}$ increase & $4.5 \mathrm{nV} \sqrt{\mathrm{Hz}}$ \\
\hline Voltage noise & $1.8 \mathrm{nV} \sqrt{\mathrm{Hz}}$ & $>10 \mathrm{nV} \sqrt{\mathrm{Hz}}$ & $0.5 \mathrm{~V} \sqrt{\mathrm{Hz}}$ \\
\hline Current noise & $1.2 \mathrm{pV} \sqrt{\mathrm{Hz}}$ & $0.5 \mathrm{fV} \sqrt{\mathrm{Hz}}$ & $\mathrm{Best}$ \\
\hline Overall performance & $\mathrm{Good}$ & Better & \\
\hline
\end{tabular}





Figure 7. Comparison between unguarded and guarded low current measurement regarding leakage current [34]

From the table-1, it is observed that CMOS op-amp is preferable to design a pico-ammeter circuit. But $1 / \mathrm{f}$ noise is a dominant source at low frequency in the sub threshold region of CMOS. In addition PMOS has a larger device area and less $1 / \mathrm{f}$ noise than that of NMOS [7].

As mentioned earlier that pico-current measurement amplifier is simply a transimpedance amplifier with large feedback resistor and high input impedance, it should be shielded along with the external input circuitry [30]. Shielding of a metallic crates to the ground or a common potential can generates stray capacitance and leakage current $[26,31]$. However, guarding is an effective way to reduce this leakage current and lowering the input stray capacitance [5] $[18,26,32]$. It should be kept in mind that the guard is not a ground, it surrounds the input trace and the ground shielding protects the guard from the external interference $[26,33]$.

Input stray capacitance can be compensated by connecting a capacitor which is parallel to the feedback resistor, as shown in figure $6[1,32]$. As it is difficult to know the value of input stray capacitance, feedback capacitor's value should be experimentally selected. Instrument response time i.e., settling time and stabilities is directly affected by this feedback capacitor. Thus, the values of feedback capacitor are as low as preferable [1].

Noise can also be generated even after shielding, if grounding is not proper. Current measuring instrument has two types of grounds. One is the signal ground or common ground and other is chassis ground or power ground. The common is the ground for the complete measuring circuit; it will affect the system's low-level measurement performance.
In contrast, the chassis ground is attached to the metal cage inclosing an instrument, mainly used for safety purpose of the user. There is no problem with connecting these grounds together. But sometimes, the power line ground can be noisy and may not be in the same level of the signal ground $[13,35]$.

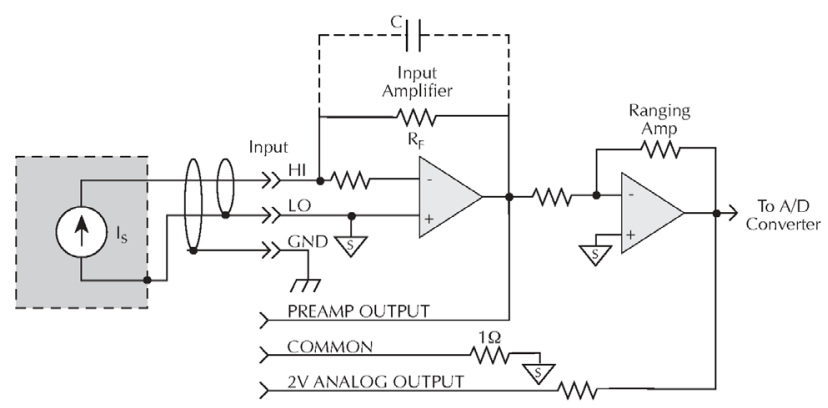

Figure 8. The feedback capacitor parallel with $\mathrm{Rf}$ and guard and grounding configuration [1].

In ultra-low current measurement, to prevent the leakage current due to poor insulating property of PCB (printed circuit board), an unconventional wiring technique can be used. In where all the leads of the components are soldered together above the board using insulated turret terminal as a support [30]. The most commonly used insulation standoff materials are fiberglass, glass, ceramic, PVC, epoxy and Teflon. Among them Teflon is generally used due to its low price. 


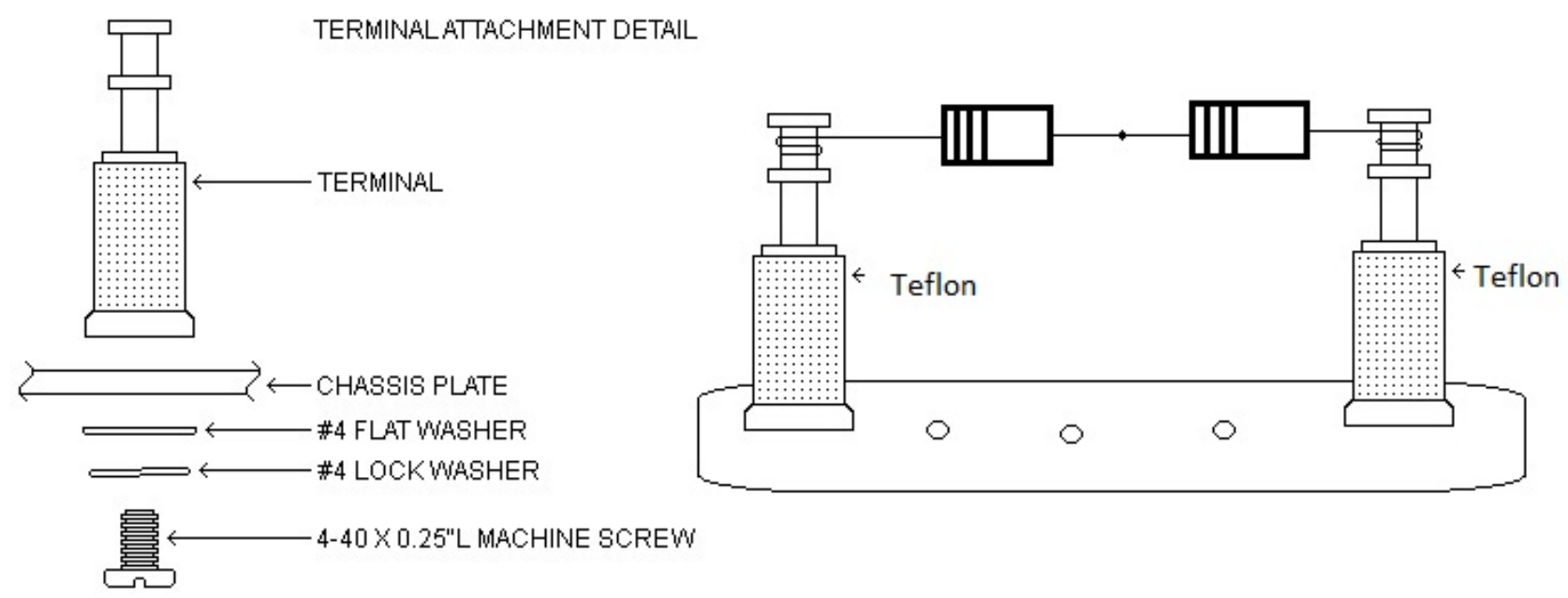

Figure 9. Turret terminal used for unconventional wiring technique

As all the components connection is made above the $\mathrm{PC}$ board, the electromagnetic influence by PCB is eliminated. This technique has an advantage of the lowest leakage, the lowest stray capacitance and overall improved current measurement performance [28]. But this technique has its limitations of commercial production.

\section{Conclusions}

In this paper, sources of noises associated with ultra-low current measurement and its minimization techniques are briefly discussed. This article is a primary help prior to design an electrometer. This paper also shows that feedback amplifier circuit is better to use as picoammeter, due to its low burden voltage and less instrument settling time over shunt amplifier. It has been observed that dust, humidity, wind, temperature and electromagnetic interference have a great impact on noise generation. Thus, it would better keep all components with circuit in a metal box in a cool and dry place. Stray capacitance and leakage current can be minimized by guarding. However, unconventional wiring techniques can reduce Piezoelectric effect. Different types of op-amp ICs have a great influence on noise generation due to leakage current and CMOS would be the best choice to overcome those problems. Triboelectric effect can be minimized by replacing ordinary cable with coax and triax cable.

\section{Acknowledgements}

The idea to write this article was developed after a nice discussion about low current measurement with Mr. Birumachi, Eng. JAEA, Japan. I would like to thank him for his cordial and clarified talk in detail about construction and measurement technique of ultra-low current.

\section{REFERENCES}

[1] O. Volynets, Measuring picoamperes, (2011) 11. https://manualzz.com/doc/7069591/measuring-picoamperes.

[2] Y. Liang, J. Chen, F.L. Duan, D. Xu, J. Wang, H. Yang, Y. Zhang, Review on Signal Detection of Low DC Current in Nanodevices and Various Sensors, Mater. Electron. Eng. 1 (2014) 1-14. doi:10.11605/mee-1-1.

[3] L.B.R. Jin Wang, Bin Kang Li, A high precision pico-amperes scale current measurement system in radiation detection, Appl. Mech. Mater. 239-240 (2013) 145-151. doi:10.

[4] N.I. Ii, N.P. Co, N. Office, I. Introduction, Monogr. Soc. Res. Child Dev. 72 (2007) 1-13. doi:10.1111/j.1540-5834.2007.00439.x.

[5] P.D.C. Current, Low Level Measurements Handbook - 7 th Edition Low Level Measurements Handbook, 7th ed., KEITHLEY, n.d.

https://www.tek.com/document/handbook/low-level-measure ments-handbook.

[6] Model 6487 Picoammeter/Voltage Source, (n.d.). https://www.rapidonline.com/pdf/1164923_da_en_01.pdf.

[7] L. Zhang, X. He, Z. Yu, Circuit Design and Verification for Ultra Low Current Sensing Amplifier Aimed at Bio-Sensor Applications, 2005 6th Int. Conf. ASIC. 1 (2005) 471-474. doi:10.1109/ICASIC.2005.1611353.

[8] Dongsoo Kim, B. Goldstein, Wei Tang, F.J. Sigworth, E. Culurciello, Noise analysis and performance comparison of low current measurement systems for biomedical applications., IEEE Trans. Biomed. Circuits Syst. 7 (2013) 52-62. doi:10.1109/TBCAS.2012.2192273.

[9] Tips for Measuring Small Currents, (n.d.). http://circuitechllar.com/cc-blog/tips-for-measuring-small-cur rents/.

[10] N.I. Switches, L.M. Resources, How to Minimize Errors for Low-Current Measurements, (2011) 2-4. http://www.ni.com/tutorial/5448/en/ 
[11] A. Keysight, Low-level current measurement using B2980A series, (2017) 1-10. http://www.keysight.com/main/editorial.jspx?cc=BD\&lc=eng $\&$ ckey $=2502168 \&$ nid $=-32788.1101300 .00 \&$ id $=2502168$.

[12] B.C. Measurements, Low Current Measurements, 2012. https://www.tek.com/document/application-note/low-currentmeasurements.

[13] S.M.U. Only, Making Ultra-Low Current Measurements with the Low-Noise Model 4200-SCS Semiconductor Characterization System Techniques for Minimizing, Earth. (n.d.).

[14] D. Kim, W. Tang, B. Goldstein, P. Weerakoon, E. Culurciello, Performance Comparison of Low Current Measurement Systems for Biomedical Applications, \{IEEE\} Int. Symp. Circuits Syst. \{ISCAS\} '10. (2010) 3469-3472.

[15] G.W.R. Adel S. Sedra, Current Conveyor Theroy And Practice, Curr. Conveyor Hist. Prog. New Results. 137 (1990). http://forum.vegalab.ru/attachment.php?attachmentid=96118 $\& \mathrm{~d}=1283849206$.

[16] A. Kumar, Design of Multi-Output Second Generation Current Controlled Conveyor, THAPAR UNIVERSITY, 2015. http://tudr.thapar.edu:8080/jspui/bitstream/10266/3482/4/348 2.pdf.

[17] A. Gupta, A Study on Current Conveyors and their Applications, J. Multi Discip. Eng. Technol. 5 (2016). www.jmdet.com/wp-content/uploads/2016/05/Paper-02.pdf.

[18] M. Circuit, L. Currents, S. Resistance, Z. Drift, O. Protection, V. Method, C. Method, S. Time, Introduction 2 Low Current Measurements 2, (n.d.).

[19] N. Instruments, Low-Current Measurement Considerations, (2009) 12-13. http://zone.ni.com/reference/en-XX/help/370384N-01/dmm/1 ow_current_measurement_considerations/.

[20] B. Goldstein, D. Kim, A. Rottigni, J. Xu, T.K. Vanderlick, E. Culurciello, CMOS Low Current Measurement System for Biomedical Applications, Iscas. 6 (2011) 1017-1020.

[21] P.W. Eugenio Culurciello, Wei Tang, Evan Joon-Hyuk Park, Brian Goldstein, Dongsoo Kim, Biomedical Circuits and Systems, Technology \& Engineering, n.d.

[22] C. Helfmeier, Charge Sensing - A Key to Improving Sensitivity in Integrated Circuit Analysis - genehmigte Dissertation, Tech. Univ. Berlin. (2015).

[23] Darren, 1/f Noise in Semiconductor Devices, (2004) 1-20. file://Users/timothyamorgan/Dropbox/Papers/Library.papers 3/Articles/2004/Darren/2004 DarrenMicrosoft Word - Final
Paper.doc.pdf\%5Cnpapers3://publication/uuid/6CA0537E-2C 94-4535-8364-A192CE0DBBC2.

[24] M. Integrated, Choosing a Low -Noise Amplifier, (2016) 1-7. https://www.maximintegrated.com/en/app-notes/index.mvp/i $\mathrm{d} / 3642$.

[25] J.L. Tucker, Optimizing Low-Current Measurements and Instruments, n.d. https://wiki.epfl.ch/carplat/documents/3100_optimizinglocurr _wp.pdf.

[26] P. Grohe, Design femtoampere circuits with low leakage, part one, (2011) 1-9.

https://www.edn.com/design/analog/4368681/3/Design-femto ampere-circuits-with-low-leakage-part-one.

[27] P. Grohe, Design Femtoampere circuits with low leakage, Part one, Edn. 56 (2011) 30-35. https://www.edn.com/design/analog/4368681/2/Design-femto ampere-circuits-with-low-leakage-part-one.

[28] P. Grohe, Design Femtoampere circuits with low leakage-Part 3: Low-current design techniques, Edn. 56 (2012) 30-35. https://www.edn.com/design/analog/4395651/Design-femtoa mpere-circuits-with-low-leakage---Part-3--Low-current-desig n-techniques.

[29] S. winder GB Clayton, Operational Amplifiers, 5th ed., Elsevier, 2003.

https://www.elsevier.com/books/operational-amplifiers/clayto n/978-0-7506-5914-7.

[30] Burr-Brown, Opa128 Operational Amplifier, 1995. http://www.ti.com/lit/ds/symlink/opa128.pdf.

[31] H. Higa, R. Onaga, N. Nakamura, A Very Low Level dc Current Amplifier Using SC Circuit: Effects of Parasitic Capacitances and Duty Ratio on Its Output, (2014) 458-466.

[32] G. Description, C. Diagrams, O. Information, LMC6001 Ultra Ultra-Low Input Current Amplifier, (2011) 1-16. papers2://publication/uuid/6CF58BDB-F89F-419A-8FCA-28 43B5F6BDE9.

[33] S.A. Dyer, Wiley Survey of Instrumentation and Measurement, in: S.A. Dyer (Ed.), Originally published: June 25, 2001, n.d.: p. 1112.

[34] A. Wadsworth, M.D. Manager, K. Technologies, Fundamentals of Low Current and Ultra-High Resistance Measurement, (2015).

[35] David G. Alciatore, Introduction to mechatronics and measurement systems, Tata McGraw-Hill Education, 2007. https://books.google.com.bd/books?id=LoZguffutukC\&prints $\mathrm{ec}=$ frontcover. 